\title{
OPTIMAL CONTENT DOWNLOADING IN VEHICULAR NETWORK WITH DENSITY MEASUREMENT
}

\author{
E. Lalitha ${ }^{1}$, D.Jayachandran ${ }^{2}$ \\ ${ }^{1}$ Student/M. Tech, KSR College of Engineering, \\ ${ }^{2}$ AP/CSE, KSR College of Engineering,
}

\begin{abstract}
The existence of Internet-connected navigation and infotainment systems is becoming a truth that will easily lead to a remarkable growth in bandwidth demand by in-vehicle users. In Examples the applications of vehicular communication proliferate, and range from the updating of road maps to the repossession of nearby points of interest, downloading of touristic information and multimedia files. This content downloading system will induce the vehicular user to use the resource to the same extent as today's mobile customers. By this approach communication-enabled vehicles are paying attention in downloading different contents from Internet-based servers. We summarize the performance limits of such a vehicular multimedia content downloading system by modeling the content downloading process as an effective problem and developing the overall system throughput with density measurement. Results highlight the methods where the Roadside infrastructure i.e., access points are working at different capabilities irrespective of vehicle density, the vehicle-to-vehicle communication.
\end{abstract}

\section{KEYWORD}

Vehicular network, Content downloading Process, Max-flow problem, Optimization, Density measurement.

\section{INTRODUCTION}

The communication-enabled vehicles are interested in downloading different multimedia contents from Internet-based servers. This system captures many of the entertainment services with effective information, such as navigation maps, news reporting service, and software updating, or multimedia content downloading. In this approach both infrastructure-to-vehicle and vehicle-tovehicle communication taken place. The major aim is to maximize the overall system throughput; we formulate a max-flow problem that accounts for several practical aspects, such as channel contention and the data transfer paradigm.

As a result, Multimedia content downloading in vehicular networks by the vehicles has received increasing attention from the research community. Initially, the availability of Infrastructure-toVehicle (I2V) communication capabilities are based on high-throughput Dedicated Short-Range Communication (DSRC) technologies, is seen as an opportunity for transfer of large data to mobile nodes that would not be possible through the existing $2 \mathrm{G} / 3 \mathrm{G}$ infrastructure, Next the availability of Vehicle-to-Vehicle (V2V) connectivity has fostered a number of proposals to make

DOI : $10.5121 /$ ijitmc.2013.1407 
use of the cooperation among vehicular users so as to improve their downloading performance. In particular, V2V connectivity based approaches are especially good when one considers that the infrastructure coverage will be mottled at initial stages, and barely seamless even at later ones.

Previous works on content downloading in vehicular networks have dealt with individual aspects of the process, such as roadside APs deployment, the performance evaluation of I2V

communication, the network connectivity, of $\mathrm{V} 2 \mathrm{~V}$ data transfer paradigms. No one has tried to deal with the problem as a whole, trying to quantify the actual potential of an I2V/V2V-based content downloading process. In order to fill such a gap, we introduce the following questions: (i) which is the maximum downloading performance achievable through DSRC-based I2V/I2V communication, in a given mobility scenario? (ii) What are the important factors that mainly determine such a downloading performance?

To answer these questions, we combine this downloading process to a mixed integer linear programming (MILP) known as max flow problem. The solution this problem results in the optimal Access point deployment over a given road layout and any possible combination of $\mathrm{V} 2 \mathrm{~V}$ and $\mathrm{I} 2 \mathrm{~V}$ data transmission.

Our framework introduces a DTNG time-invariant graph. We do not undertake the contacts between mobile nodes to be same but allow them to access directly, and also report the presence of roadside infrastructure and channel contention. Such an approach allows us to significantly enhance the AP deployment over the given road layout, since we maximize the overall throughput and also provide the optimal solution instead of an approximation.

At the result, the access point or relay shows the vehicle capability prior and sends the corresponding low quality or high quality file. This achieves the vehicle to receive the proper file resource. Vehicle density is calculated based on previous temporal changes and the new vehicle density is calculated. The access points' capabilities are adjusted so that it works more in high vehicle density environment and works less in low vehicle density environment.

This paper is organized as follows: Section II describes the previous work, while Section III discusses contribution of work. In Section IV, we build the system model and assumption, while we generate the Dynamic Network topology graph in Section V and we formulate the max-flow problem in Section VI, Results, derived in the design guidelines described in Section VII. In section VIII, we evaluate the vehicle density based data downloading. Section IX describes Security issues; finally section $\mathrm{X}$ summarizes our major findings and point out direction of future work.

\section{RELATED WORK}

The authors U. Paul, M.M. Buddhikot, A.P. Subramanian, and S.R. Das were stated that the complete measurement analysis of network resource deployment and the subscriber activities using a large-scale data set collected within a nationwide $3 \mathrm{G}$ cellular network. The data set keeps close to more number of subscribers over thousands of base stations. They also examine the capability of network resources which can be used by different subscribers as well as by different applications. They also find out the traffic in vehicular network from the point of view of the base stations and analyze the temporal and spatial variations in different kinds of the vehicular 
network. In order to address such coverage uncertainties the authors Z. Zheng, P. Sinha, and S. Kumar were given a idea about new the alternating coverage for mobile users, called $\alpha$-coverage, and examined how such coverage can be attained by systematic deployment of more APs to create an efficiently scalable infrastructure. In other way, a deployment of APs involved in $\alpha$ coverage to a network topology, if the road with length $\alpha$ on the given network resource meets with at least one AP in that resource. The authors Z. Lu, Z. Zheng, P. Sinha, and S. Kumar were also stated that with increasing popularity of media enabled devices; the need for high data-rate services for mobile users is obvious. Large-scale Wireless LANs (WLANS) can offer such a service, but they are very expensive to deploy and maintain. The above results not make the grade to provide any throughput assurance to a vehicular user; it can only provide opportunistic services to them.

\section{MY CONTRIBUTION}

The density measurement in vehicular network my contributions to this problem are as follow:

- The access point or relay tracks the vehicle capability prior and sends the corresponding low quality or high quality file. This achieves the vehicle to receive the proper file resource

- Vehicle density is calculated based on previous temporal changes and the new vehicle density is calculated.

- The access points' capabilities are adjusted so that it works more in high vehicle density environment and works less in low vehicle density environment.

- Vehicle density based download scenario is applied to Access Points.

Proposed methods where the Roadside infrastructure i.e., access points are working at different capabilities irrespective of vehicle density.

\section{SYSTEM MODEL AND ASSUMPTIONS}

\subsection{Network Model}

We create a network composed of fixed roadside APs and vehicular users, where some of them are downloader's. They are interested in downloading multimedia content from the Internet through the APs. We consider the general case in which every downloader may be interested in different content. They can either use relays or establish direct connectivity with APs. In particular, we consider the following data transfer paradigms:

Direct transfers, a direct communication between an AP and a downloader. This shows the typical way how the mobile users communicate with the infrastructure as in today's wireless networks;

Connected forwarding, the result shows communication made through one or more vehicles that create a multi hop path between an AP and a downloader. This is the conventional approach to traffic delivery in ad hoc networks; 
Carry-and-forward, the communication made through one or more vehicles that store and carry the data, and delivering them either to the target downloader or to another relay which meet such downloader sooner.

Our approach allows us to processing a road layout and an associated vehicular mobility trace, so as to build a time expanded graph that represents the temporal network evolution (Sec. V). By using this graph, we formulate a max-flow problem; the solution of this problem matches our goals.

\section{DYNAMIC NETWORK TOPOLOGY GRAPH}

Dynamic network topology graph (DNTG) generate a from a different vehicular mobility trace in network topology, considering that on the corresponding road layout there are: (i) a set of $\mathrm{A}$ candidate locations $\left(a_{i}, \mathrm{i}=1, \ldots, \mathrm{A}\right)$ where APs could be placed (ii)a set of V vehicles $\left(v_{i}, \mathrm{i}=1\right.$, $\ldots, \mathrm{V}$ ) travel over the road layout (iii) a set of $\mathrm{D}$ vehicles that wish to download data from the APs.

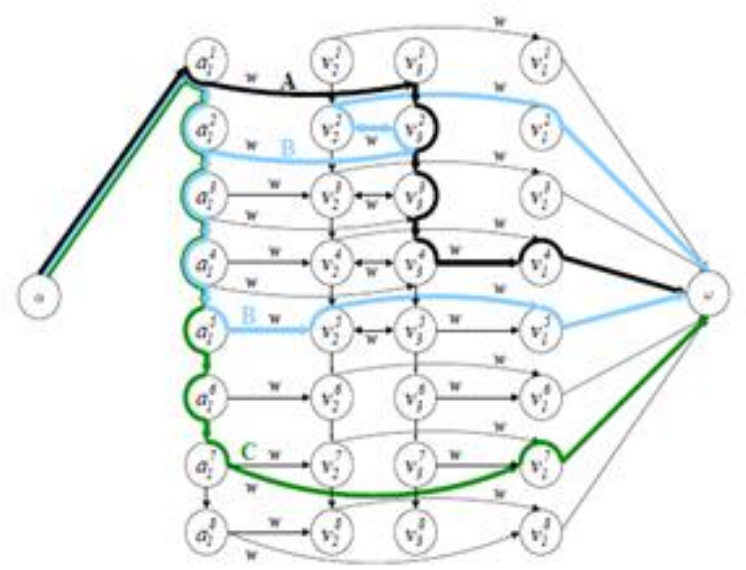

Figure1. A sample DNTG, with one Access point A and three vehicles v1, v2 \& v3, the vehicle (v1) is a downloader while the others (v2, v3) can act as relays. In the above graph, we show up paths that are agent of the carry and-forward (A), connected forwarding (B), and direct transfer (C) paradigms.

The major aim of this topology graph is to model all possible ways through which data can flow from either direct APs to the downloader's or possibly via relays. With known mobility trace, we identify the contact events between any pair of nodes such as V2I/V2V.

Each contact event is characterized by:

- Link quality, The quality of the link between the two nodes; specifically, the achievable data transfer rate at the network layer, which depends on the distance between the possible two nodes

- The contact starting time, The time at which the link between the two nodes is established or already established link that has quality level with new value; 
- A contact ending time, the time at which, the quality level of link has changed when the link is removed or discarded

The time interval between any two contact events in the network is called frame. Within a frame the network is static, i.e., the link quality levels do not change which means no link is created or removed or no contact event is established. We denote the number of frames by $\mathrm{F}$, and the duration of the frame is $\mathrm{k}(1 \leq \mathrm{k} \leq \mathrm{F})$ by $\mathrm{k}$; also, all constant contact events during each frame $\mathrm{k}$ are said to be active in that same frame. The vehicle $V_{i}$ shares in the network at frame $\mathrm{k}$ is represented by a vertex $V_{i}^{k}(1 \leq \mathrm{i} \leq \mathrm{V})$ in the above graph, where as each candidate AP location $A_{i}$ is mapped within each frame $\mathrm{k}$ onto a vertex $A_{i}^{k}(1 \leq \mathrm{i} \leq \mathrm{A})$. We denote by $V^{k}$ and $A^{k}$. The subset of vertices representing the downloader's that existing in the network at frame $\mathrm{k}$ denoted by $D^{k} C V^{k}$. All non-downloader vehicles in the given road layout denoted by $R^{k}=V^{k} \backslash D^{k}$ can act as relays, according to the data transfer paradigms is shown through graph.

Within each frame $\mathrm{k}$, a directed edge $\left(V_{i}^{k} \in V_{j}^{k}\right)$ exists fromvertex $V_{i}^{k} \in R^{k}$ to vertex $V_{j}^{k} \in V^{k}$ if a contact between thenon-downloader vehicle $V_{i}$ and another vehicle $V_{j}$ is active during that frame. Each edge of this frame type is associated with a weight $\mathrm{w}\left(V_{i}^{k}, V_{j}^{k}\right)$, equal to the rate of that corresponding contact event. The set having such edges is denoted as $L_{v}^{k}$.

Similarly, a directed edge $\left(A_{i}^{k}, V_{j}^{k}\right)$ comes from vertex $A_{i}^{k} \in A^{k}$ to vertex $V_{j}^{k} \in V^{k}$ if a contact between the candidate AP Ai and the vehicle $V_{j}$ is active during frame $\mathrm{k}$. These edges are related with weights $\mathrm{w}\left(A_{i}^{k}, V_{j}^{k}\right)$, equivalent to the contact event rate, and their set is defined as $L^{k}$ a. A directed edge $\left(V_{i}^{k}, V_{i}^{k+1}\right)$ is also drawn from any vertex $V_{i}^{k} \in R^{k}$ to any vertex $V_{i}^{k+1} \in R_{i}^{k+1}$, for $1 \leq \mathrm{k} \leq \mathrm{F}$. While the edges in $L_{v}^{k}$ and $L_{a}^{k}$ represent transmission opportunity, those of the form $\left(V_{i}^{k}, V_{i}^{k+1}\right)$ model the possibility that a nondownloader vehicle $V_{i}$ physically carries some data during its association from frame $\mathrm{k}$ to frame $\mathrm{k}+1$. However, dealing with vehicular nodes as conflicted to resource-constrained hand-held devices, we take the weight of such edges to be assume on an infinite value. A directed edge $\left(A_{i}^{k}, A_{i}^{k+1}\right)$ of infinite weight is held between two any vertices or nodes representing the same candidate AP at two successive frames, i.e., from $A_{i}^{k} \in A^{k}$ to $A_{i}^{k+1} \epsilon A^{k+1}(1 \leq \mathrm{k} \leq \mathrm{F})$. We will refer to the edges of the kind $\left(V_{i}^{k}, V_{i}^{k+1}\right)$ or $\left(A_{i}^{k}\right.$, $\left.A_{i}^{k+1}\right)$ as intra-nodal.

Finally, in order to originate a max-flow problem over the DNTG, we introduce two virtual nodes, denoted as $\propto$ and $\omega$, respectively representing the source and destination of the total flow of the graph. Then, the graph is finished with infinite weight edges $\left(\propto, a_{1}^{i}\right)$, from $\propto$ to any vertex $a_{1}^{i} \in a_{1}$, and $\left(V_{i}^{k}, \omega\right)$, from any vertex $V_{i}^{k} \in D_{k}$ to $\omega, 1 \leq \mathrm{k} \leq \mathrm{F}$.

The DNTG is therefore a weighted directed graph, representing the network topology development over time. An example of DNTG is given in Fig. 1, representing one AP and three vehicles $\mathrm{v} 1, \mathrm{v} 2, \& \mathrm{v} 3$, with $\mathrm{v} 1$ is considered as a downloader and v2, v3 vehicles are acting as relays. To minimize the graph size, in the above example we assume the possible network-layer rate $\mathrm{w}$ to be constant during the complete lifetime of a link. Note that the graph allows the capture of all the data transfer paradigms previously discussed. It is possible to identify paths in the graph that correspond to (1).In path $\mathrm{C}$ direct download from the Access point to the downloader. (2).In path B connected forwarding through 3-hops (frame 2) and 2-hops (frame 5) and (3).In path A carry-and-forward through the movement in time of the relay vehicle $\mathrm{v} 3$. 


\section{THE MAX-FLOW PROBLEM}

With the above topology graph, our next step is to formulate the optimization problem the main goal of this problem is to maximize the flow from $\propto$ to $\omega$, i.e., the total amount of data downloaded by the downloader's. Denoted by x $\left(V_{i}^{k}, \omega\right)$ the traffic flow over an edge connecting two generic vertices, our intention can be expressed as:

$$
\max \sum_{k=1}^{F} \sum_{V_{i}^{k} \in D^{k}} x\left(V_{i}^{k}, \omega\right) .
$$

The max-flow problem needs to be solved taking into account several constraints such as, maximum number of APs that can be activated, channel access, non negative flow and flow conservation. We detail such constraints below.

\section{A. Constraints}

Non-negative flow and flow conservation: the flow on each existing edge in DNTG must be greater than or equal to zero. Also, for any vertex in the graph, the amount of ingoing flow into the vertex must equal the amount of outgoing flow.

Channel access: In view of the fact that we consider an IEEE 802.11-based MAC scheme with RTS/CTS and we assume unicast transmissions, two or more of the following events cannot take place simultaneously for a tagged vehicle, and the time duration of each frame must be shared among the tagged vehicle:

1) The vehicle transmits to a neighboring vehicle;

2) A neighboring vehicle receives from any relay;

3) The vehicle receives from a neighboring relay;

4) A neighboring relay transmits to any vehicle;

5) The vehicle receives from a neighboring AP;

6) A neighboring AP transmits to any vehicle.

In the above states we have only consider the total amount of data carried by each flow. Due to the use of RTS/CTS in 2) a neighboring vehicle receiving data is accounted, considering that the sub cases are: 1) is a subcase of 2);3) is a subcase of 4); 5) is a subcase of 6), for the generic vertex $V_{i}^{k} \in V^{k}$ and for any frame $\mathrm{k}$, we have:

$$
\begin{aligned}
& \sum_{\substack{V_{j}^{k} \varepsilon R_{v}^{k} V_{m}^{k} \in V_{m}^{k} \in V^{k} \\
\left(V_{j}^{k}, V_{m}^{k}\right) \in L_{v}^{k}}} 1\left[\left(v_{m}^{k}, v_{j}^{k}\right)\right] \frac{x\left(v_{j}^{k}, v_{m}^{k}\right)}{x\left(v_{j}^{k}, V_{m}^{k}\right)} \\
& +\sum_{\substack{V_{j}^{k} \varepsilon R^{k} V_{m}^{k} \in V_{m}^{k} \in V^{k} \\
\left(V_{j}^{k}, V_{m}^{k}\right) \in L_{v}^{k}}} 1\left[\left(v_{j}^{k}, v_{i}^{k}\right)\right] \frac{x\left(v_{j}^{k}, v_{m}^{k}\right)}{x\left(v_{j}^{k}, v_{m}^{k}\right)}+\sum_{\substack{v_{j}^{k} \varepsilon R^{k} V_{m}^{k} \in V_{m}^{k} \in V^{k} \\
\left(V_{j}^{k}, V_{m}^{k}\right) \in L_{v}^{k}}} 1\left[\left(a_{m}^{k}, v_{j}^{k}\right)\right] \frac{x\left(a_{j}^{k}, v_{m}^{k}\right)}{x\left(a_{j}^{k}, v_{m}^{k}\right)} \leq \tau^{k} \\
& \qquad
\end{aligned}
$$


If the specified edge exists then the indicator function is equal to 1 otherwise it is 0 . In addition, for each candidate AP location, we have that the total transmission time of each candidate during each frame k cannot exceed the frame duration. Thus, for any k and $A_{j}^{k} \in A^{k}$, we have the equation as:

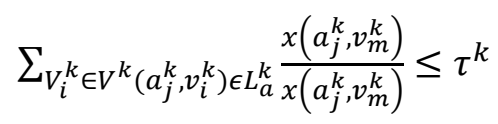

The above constraints allow a vehicle under AP coverage area to utilize either I2V or V2V communication within the same frame. Next, we consider a vehicle under the coverage of either one AP is not connected to work in ad hoc mode, which means the communication with other vehicle is not possible. Then, for every frame $\mathrm{k}$ and $V_{j}^{k} \in R^{k}, V_{m}^{k} \in v^{k}$ such that $\left(V_{j}^{k}, V_{m}^{k}\right) \in L_{v}^{k}$, the following constraint holds:

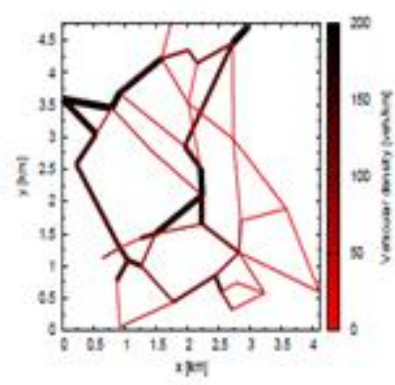

(a) Vebicular density over space

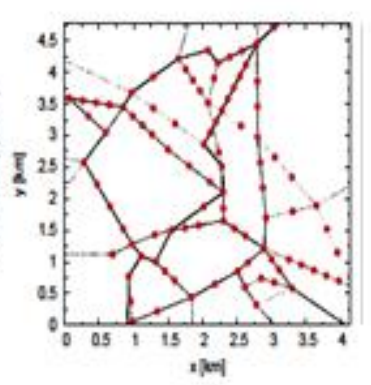

(b) AP candidate locations

Figure2. Simulation scenario: (a) road layout and average density of vehicles computed over a whole day; (b) giving out of the AP candidate locations over the road layout.

$$
\mathrm{x}\left(v_{j}^{k}, v_{m}^{k}\right) \leq\left(1-\max _{\substack{a_{i}^{k} \in A^{k} \\\left(a_{j}^{k}, v_{i}^{k}\right) \epsilon L_{a}^{k}}}\left\{y_{i}\right\}\right) w\left(V_{j}^{k}, V_{m}^{k}\right) \tau^{k}
$$

Where $y_{i}, \mathrm{i}=1 \ldots \mathrm{A}$, are Boolean variables, If the candidate $\mathrm{AP} \mathrm{Ai}$ is activated the value is 1 and the value becomes 0 otherwise.

Maximum number of active APs: The final set of constraints imposes that no more than one candidate APs are selected, through the variables yi. Then, for any i, we can write:

$$
y_{i} \in\{0,1\} ; \sum_{i=1}^{A} y_{i} \leq \hat{A} ; x\left(\propto . a_{i}^{1}\right) \leq M y_{i}
$$

Where $\mathrm{M} \epsilon \mathrm{R}$ is a randomly large positive constant.

\section{DERIVING DESIGN GUIDELINES}

We influence the problem formulation obtained in the previous section to illustrate which factors concern the most in content downloading process in vehicular networks and to provide realistic hints for the design of a system. We consider a real-world road topology, covering an area of 10 
$\mathrm{km} 2$ in the urban area. The vehicular mobility trace in the region has been synthetically generated at urban area, through a multi-agent microscopic traffic simulator. In Fig. 2(a), we describe the road layout which explains the different traffic methodology observed over each road layout.

We consider a traditional VANET technology penetration rate, which means that only a fraction of the vehicles in the network, namely $20 \%$, is equipped with a communication interface or communication device and is ready to participate in the content downloading process, either as relays or as downloader's. Also, the number of vehicular downloader's that concurrently request content is assumed to be $1 \%$ of the vehicles participating in the network. AP locations are selected along the roads such that the distance between two adjacent APs is slightly equal to 150 $\mathrm{m}$, resulting in 92 candidate locations, shown in Fig. 2(b). The value of the achievable networklayer rate between any two nodes is attuned according to the distance between them. We bounded the maximum node transmission range to 200m; this distance allows the establishment of a reliable communication in $80 \%$ of the cases.

Since we make use of a realistic mobility model, in each road topology the intensity of the vehicular traffic varies depending on the road segment and time period of the day. In Fig. 3a, 3b, and $3 \mathrm{c}$, we describe the road layout of the urban, village, and suburban village environments, stress the different traffic volumes observed over each road segment: Thicker, highlighted density segments

identify the roads layout characterized by higher vehicular density. As far as vehicular traffic variations in given road layout is concerned, we consider only time periods corresponding to the density of vehicles.

In the urban, village, and suburban road layout traces, each enduring about 6 hours, this leads to an average density of $90,62.5$ and 33.5 veh $\mathrm{km}$, respectively. The value of the attainable network-layer rate between every two nodes is adjusted according to the distance between them. To this end, we refer to the 802.11a experimental results that obtain the values shown in Fig. 3d, and we use them as samples of the achievable network-layer rate. Note that we ends up the maximum node transmission range to $200 \mathrm{~m}$, because, this distance allows the establishment of a reliable communication in 80 percent of the cases.

Given that $A^{\wedge}$ locations have to be activated, the result of the max-flow problem in Sec. 4 provides the AP deployment that maximizes the aggregate download throughput. We benchmark the performance of our optimal Max-flow strategy against the following AP deployment policies:

Random: According to a uniform distribution, $\mathrm{AP}^{\wedge}$ locations are randomly selected among the candidate.

Crowded: It selects the $\mathrm{AP}^{\wedge}$ locations whose coverage area exhibits, above the highest vehicular density;

Contact: It picks up the $\mathrm{AP}^{\wedge}$ locations that maximize the addition of the contact opportunities between vehicles and APs. 


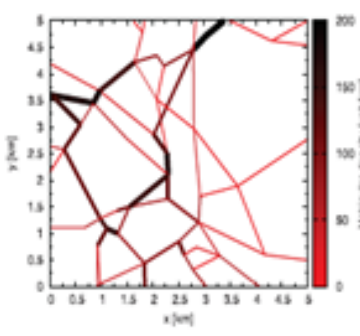

(a) Urban: traffic over space

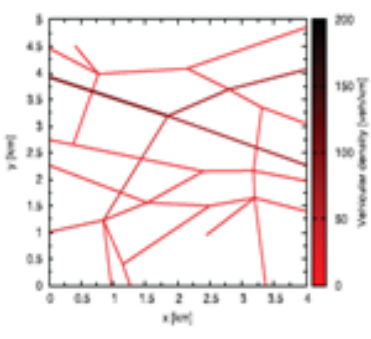

(b) Village: traffic over space

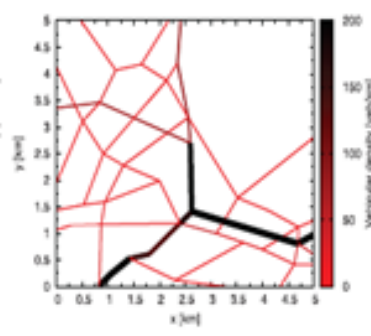

(c) Suburban: traffic over space

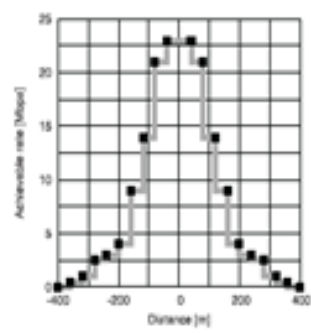

(d) Network-layer rate

Figure3. Road layout in the (a) urban, (b) village, and (c) suburban scenarios, and achievable network-layer rate characterization as a function of distance (d).

Particularly, for each vehicle, the contact opportunity is expressed as the fraction of the road section lengths traveled while under coverage of at least one AP. Once the active AP locations in the given road layout are determined according to any one of the above three policies, they are used in the max-flow problem formulation to secure the values of the binary variables yi. Since the system throughput is obtained as the result of the max-flow problem with the preferred AP locations yi, the results we show represent the preeminent performance one can achieve with each deployment strategy. Fig. 3 shows the average content downloader throughput for different deployment strategies, with the function of the number of active APs A*.

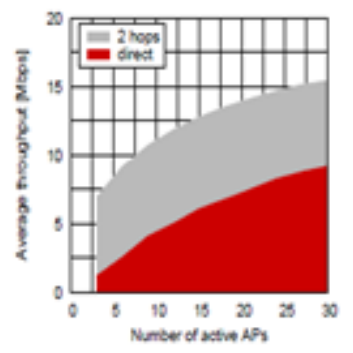

(a) 2-bop limit

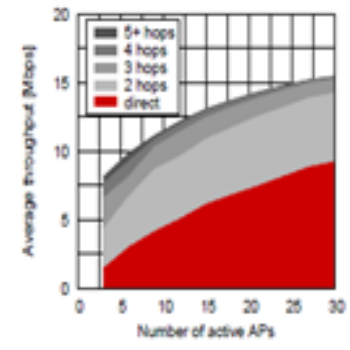

(b) unlimited

Figure4. Partition of the average downloader throughput with respect to the number of relays between AP and downloader (Max-flow deployment strategy)

In order to demonstrate the absolute result of the throughput figures reported above, we focus on the Max-flow deployment strategy and look at the number of hops that data go through before reaching their destination. In Fig. 4(a), the hop limit is set to 2 which means the number of relays between APs is 2, thus the plot in the above graph describes the portion of the average per downloader throughput is due to direct data transfers and which is instead reached using one relay, when the number of deployed APs is small then the last hop largely dominates the previous hop. As the existence of APs becomes more pervasive, direct transfers paradigm are clearly more frequent. However, it is most important to observe that the amount of data downloaded through one relay remains constant, even when 25 APs covering $50 \%$ of the road layout are deployed. The proportion of throughput achieved through direct and multi-hop data transfers does not change when the boundary on the number of allowed hops is removed, in Fig. 4(b). There, we can also note the small contribution due to transfer's over 3 or more hops, specifically for $\hat{A} \leq 10$. Finally, 
the comparison between Fig. 4(a) and Fig. 4(b) shows the complexity due to the use of more than one relay at a time can be eliminated without significant destruction. To summarize, we illustrate the following conclusions:

- Traffic relaying, through either connected forwarding or carry-and-forward, can considerably increase the average per-downloader throughput, even when the road layout is covered by more APs;

- Multi-hop data transfers involving more than one relay are less beneficial to the content downloading process.

\section{VEHICLE DENSITY BASED ACCESS POINT DATA DOWNLOADING}

In addition, the access point or relay tracks the vehicle capability prior and sends the corresponding low quality or high quality file. This achieves the vehicle to receive the proper file resource.

Vehicle density is calculated based on previous temporal changes and the new vehicle density is calculated. The access points' capabilities are adjusted so that it works more in high vehicle density environment and works less in low vehicle density environment.

\section{SECURITY ISSUES}

\subsection{Digital signatures as a building block}

The message authenticity is necessary to protect VANETs from outsiders. But since safety messages will not contain any sensitive information confidentiality is not required. In this system, the exchange of safety messages by vehicles in a VANET needs authentication of message but no need for encryption of such message. Symmetric authentication mechanisms usually encourage less overhead per message than their asymmetric counter parts. In the VANET setting, safety messages are typically standalone and should be sent to receivers as quick as possible so the digital signatures are a better choice. In fact, a preface handshake is not suitable and actually creates more overhead. In addition, with the huge amount of network participants and the irregular connectivity to authentication servers, a PKI (Public Key Infrastructure) mechanism is the most suitable method for implementation of message authentication.

\subsection{Estimation of the signature size}

As we intend using a PKI for supporting security in VANETs, it is significant to choose a Public Key Cryptosystem (PKCS) with a tolerable implementation overhead in the vehicular network. According to DSRC, safety messages are sent with a periodicity of 100 to $300 \mathrm{~ms}$. this inflict an upper bound on the processing time overhead; this overhead is shown below:

$\mathrm{T}_{\mathrm{oh}}(\mathrm{M})=\mathrm{T}_{\mathrm{sign}}(\mathrm{M})+\mathrm{T}_{\mathrm{tx}}\left(\mathrm{M} \mid \operatorname{Sig}_{\mathrm{PrKv}}[\mathrm{M}]\right)+\mathrm{T}_{\text {verify }}(\mathrm{M})$

Where $\mathrm{T}_{\mathrm{sign}}(\mathrm{M}), \mathrm{T}_{\mathrm{tx}}(\mathrm{M})$, and $\mathrm{T}_{\text {verify }}(\mathrm{M})$ are the necessary time durations to sign, transmit, and verify a message $M$, respectively; $\operatorname{Sig}_{\mathrm{PrKv}_{\mathrm{r}}}[\mathrm{M}]$ is the signature of $M$ and also includes the CA's 
certificate of the signing key by the sending vehicle V. The above expression shows the three factors that affect the choice of a particular PKCS: (1) the execution speeds of the signature generation (2) the verification operations, and (3) the sizes of key, signature, and certificate.

Since the actual size of encrypted messages is between 100 and 200 bytes, before being signing, the message is hashed. The overhead is almost constant for a given cryptosystem.

Hence, it is possible to evaluate different options at least relatively to each other .In fact, there are more number of candidate PKCS for implementing the PKI in a VANET. To ensure the future security of the PKCS, and taking into account the deployment schedule of DSRC.

Table 1: Size and transmission time of PKCS

\begin{tabular}{|c|c|c|}
\hline PKCS & Sig size(bytes) & $\mathrm{T}_{\mathrm{tx}}(\operatorname{Sig})(\mathrm{Ms})$ \\
\hline RSA & 256 & 0.171 \\
\hline ECDSA & 28 & 0.019 \\
\hline NTRU & 197 & 0.131 \\
\hline
\end{tabular}

Table 2: Comparison of signature generation and verification times on a memory-constrained Pentium II $400 \mathrm{MHz}$ workstation

\begin{tabular}{|c|c|c|}
\hline PKCS & Generation(ms) & Verification(ms) \\
\hline ECDSA & 3.255 & 7.617 \\
\hline NTRU & 1.587 & 1.488 \\
\hline
\end{tabular}

We list records for public key and signature sizes:

1. RSA Sign: the key size and signature sizes are large (256 bytes).

2. ECC (Elliptic Curve Cryptography): it is smaller than RSA (28 bytes), slower in verification but faster in signing.

3. NTRU Sign 4: the key size is lies between the RSA and ECC (197 bytes), but in both signing and verification. it is much faster than the RSA and ECC

In DSRC the least data rate is $6 \mathrm{Mbps}$ (for safety messaging data rate is typically $12 \mathrm{Mbps}$ ), the transmission overhead (at $12 \mathrm{Mbps}$ ) is acceptable, and these two options are shown in Table 1 and Table 2 gives approximate execution times of signature generation and verification for ECDSA (Elliptic Curve Digital Signature Algorithm) and NTRU Sign. These figures in the table should be taken only as suggestive for the specific platform such as Pentium II $400 \mathrm{MHz}$ with memory constraints.

In conclusion, we can observe that in terms of performance, ECDSA and NTRU outperform RSA. Compared to each other, the advantage of ECDSA is its small and economically designed; whereas NTRU's is more speed than ECDSA. The result should depend on case-specific evaluations. 
International Journal of Information Technology, Modeling and Computing (IJITMC) Vol.1,No.4,November 2013

\section{CONCLUSION}

We examined the main factors affecting the performance of content downloading process in vehicular networks, by formulating and solving a max-flow problem over a time extended graph representing a realistic vehicular trace.

The important results in our system are as follows:

- Our major ideas are that a density-based AP deployment yields performance close to the optimum result, and that multi-hop traffic delivery is valuable, although the gain is negligible beyond 2 hops from the AP.

- The access points' capabilities are adjusted so that it works more in high vehicle density environment and works less in low vehicle density environment.

To our best knowledge, this paper addressing the security of vehicular networks in a efficient and quantified way.

In terms of future work, we aim to further develop this proposal. In particular, we plan to explore in more detail the respective merits of key distribution by the manufacturers or by legislative bodies; we will also going to carry out additional numerical evaluations of the solutions.

\section{REFERENCES}

[1] M.Francesco.C. Claudio,C. Carla-Fabiana and F. Marco, "Optimal content downloading in vehicular networks," proc. IEEE INFOCOM, July 2013.

[2] U.Paul, A.P. Subramanian, S.R. Das and M.M. Buddhikot, "Understanding Traffic Dynamics in Cellular Data Networks,” Proc. IEEE INFOCOM, Apr. 2011.

[3] K.Pentikousis, M. Palola, M. Jurvansuu, and P. Perl, "Active good put measurements from a public 3G/UMTS network," IEEE Communica-tions Letters, vol. 9, pp. 802-804, 2005.

[4] P.R eichl, M. Umlauft, J. Fabini, R. Lauster, and G. Pospischil, "Project WISQY: A measurementbased end-to-end application-level performance comparison of $2.5 \mathrm{G}$ and $3 \mathrm{G}$ networks," in Proc. Fourth Ann. Wireless Telecomm. Symp (FTS), 2005.

[5] K.Mattar, A. Sridharan, H. Zang, I. Matta, and A. Bestavros, "TCP over C DMA2000 networks: A cross-layer measurement study," in Proc. PA M , 2007

[6] D Willkomm, S. Machiraju, J. B olot, and A. Wo lisz, "Primary users in cellular networks: A largescale measurement study," in Proc. DySPAN, 2008.

[7] R.Keralapura, A. Nucci, Z.-L. Zhang, and L. Gao, "Profiling users in a 3 G network using hourglass co-clustering," in Proc. ACM MobiCom, 2010.

[8] VeriWise Asset Intelligence. http://www.ge.com/equipmentservices/ asse intelligence/.

[9] A.Balasubramanian, R. Mahajan, A. Venkataramani, B. N. Levine, and J. Zahorjan. Interactive Wi-Fi Connectivity for Moving Vehicles. In Proc. of ACM SIGCOMM, Sept. 2008.

[10] V.Bychkovsky, B. Hull, A. K. Miu, H. Balakrishnan, and S. Madden. A Measurement Study of Vehicular Internet Access Using In Situ Wi-Fi Networks. In Proc. o f ACM MOBICOM, Sept. 2006.

[11] J.Eriksson, H. Balakrishnan, and S. Madden. Cabernet: A Wi-Fi-Based Vehicular Content Delivery Network. In Proc. of ACM MOBICOM, Sept. 2008.

[12] V.Navda, A. P. Subramanian, K. Dhanasekaran, A. Timm-giel, and S. R. Das. MobiSteer: Using Steerable Beam Directional Antenna for Vehicular Network Access. In Proc. o f MOBISYS, 2007.

[13] J.Ott and D. Kutscher. Drive-thru Internet: IEEE 802.11b for "Auto-mobile" Users. In Proc. of I NFOCOM , Mar. 2004. 
International Journal of Information Technology, Modeling and Computing (IJITMC) Vol.1,No.4,November 2013

[14] Z.Zheng S. Kumar, and P. Sinha,. "Alpha Coverage: Bounding the Interconnection Gap for Vehicular Internet Access." Technical Report OSU-CISRC-1/09-TR03, ftp://ftp.cse.ohio-state.edu/pub/techreport/2009/TR03.pdf, 2009.

[15] Z.Zheng, Z. Lu and P. Sinha, "Maximizing the Contact Opportunity for Vehicular Internet Access," Proc. IEEE INFO-COM, Mar. 2010

[16] Google Wi-Fi. Google's Mountain View Wi-Fi Network. http://wifi.google.com/.

[17] Taking Wireless to the Max. Business Week (business-week.com/go/techmaven), pages 101-102, Nov. 2008.

[18] WiMax.com FAQ. http://www.wimax.com/education/faq/, 2008

[19] U.S.Census Bureau - TIGER/Line http://www.census.gov/geo/www/tiger/.

[20] VeriWise Asset Intelligence. http://www.ge.com/equipmentservices/ asse intelligence/.

[21] M.Fiore and J.M. Barcelo-Ordinas, "Cooperative Download in Urban Vehicular Networks," Proc. IEEE Sixth Int'l Conf. Mobile Ad Hoc and Sensor Systems (MASS), Oct. 2009

[22] F.Aidouni, C. Magnien , M. Latap , "Ten weeks in the life of an eDonkey server", Hot-P2P'09, Rome, Italy, May 2009.

[22] K.Fall, "A delay-tolerant network architecture for challenged Internets", Proc. ACM Sigcomm'03, Karlsruhe, Germany, August 2003.

[23] S.Keshav, D. Hadaller, S. Agarwal, and T. Brecht "Vehicular Opportunistic Communication under the Microscope," Proc. ACM MobiSys, June 2007 